\title{
Editorial: Advancing Our Understanding of Structure and Function in the Brain: Developing Novel Approaches for Network Inference and Emergent Phenomena
}

\author{
Chris G. Antonopoulos ${ }^{1 *}$, Nicolás Rubido ${ }^{2,3}$, Antonio M. Batista ${ }^{4}$ and Murilo S. Baptista ${ }^{5}$ \\ ${ }^{1}$ Department of Mathematical Sciences, University of Essex, Colchester, United Kingdom, ${ }^{2}$ Aberdeen Biomedical Imaging \\ Centre, University of Aberdeen, Aberdeen, United Kingdom, ${ }^{3}$ Instituto de Física de Facultad de Ciencias, Facultad de Ciencias, \\ Universidad de la República, Montevideo, Uruguay, ${ }^{4}$ Department of Mathematics and Statistics, State University of Ponta Grossa, \\ Ponta Grossa, Brazil, ${ }^{5}$ Department of Physics, Institute for Complex Systems and Mathematical Biology (ICSMB), University of \\ Aberdeen, Aberdeen, United Kingdom
}

Keywords: biophysics, network inference, synchronisation, structure and function in the brain, complex systems, emergent phenomena

Editorial on the Research Topic

\section{OPEN ACCESS}

Edited by:

Silvina Ponce Dawson, University of Buenos Aires, Argentina

Reviewed by: Luis Diambra, National University of La Plata, Argentina

*Correspondence: Chris G. Antonopoulos canton@essex.ac.uk

Specialty section: This article was submitted to Biophysics,

a section of the journal

Frontiers in Physics

Received: 04 November 2020 Accepted: 10 November 2020 Published: 15 January 2021

Citation:

Antonopoulos CG, Rubido N, Batista AM and Baptista MS (2021) Editorial: Advancing Our

Understanding of Structure and Function in the Brain: Developing Novel Approaches for Network Inference and

Emergent Phenomena.

Front. Phys. 8:626093.

doi: 10.3389/fphy.2020.626093
Advancing Our Understanding of Structure and Function in the Brain: Developing Novel Approaches for Network Inference and Emergent Phenomena

Complex systems are ubiquitous in nature and are the subject of intense research in the last decades [1]. A complex system is composed of a large number of non-trivially interacting components whose collective behavior cannot be determined from the behavior of the components [2]. Many real-world systems can be modeled as complex, such as stock markets, the Internet and the brain [3].

In the brain, a massive number of microscopic components (neurons or cortical areas) interact in nonlinear ways, where important information resides in the relationships among them and not necessarily within their individual dynamics. Hence, studying the dynamics of these components without knowing how they are interconnected does not allow for the understanding of the behavior of the brain as a whole. Furthermore, connectivity is often unknown and difficult to infer due to large system-sizes and multiple time and spatial scales. This poses significant challenges and open questions.

The work in this Research Topic advances our understanding on complex systems at large and on the inner workings of the brain. The reader will find novel analytical and computational results on these fields as well as the mathematical-models pertaining to complex systems and network neuroscience.

In the first paper by Lassa Ortiz et al., the open problem of the nervous system representation of a motor program is addressed. In birdsong production, it has been proposed that some special temporal instances are preferentially represented in the cortex. The authors computed these temporal instances for two species and reported which of them is better suited to test the proposed coding and alternative models, against data.

The mechanisms underlying effective propagation of high intensity information over a background of irregular firing and response latency in cognitive processes remain unclear. To address this open problem, the author in He proposes a synchronous spiking cortical column propagation integration circuit (SSCCPI). Using numerical simulations, it was demonstrated that SSCCPI circuits provide a possible mechanism of effective signal propagation in cortical networks. 
Interactions between different temporal scales of the information flow in complex networks were studied in Sotero et al. and were found to be stronger in scale-free than in Erdős-Rényi networks. It was also found that phaseamplitude coupling decreased in subjects with Alzheimer's disease compared to healthy controls. These results suggest a link between cognitive impairment and multi-scale information flow in the brain.

Imagined activities can be a cognitive basis for creative thinking. However, it is still unknown how they are related to the architecture of the brain. The authors in Baravalle et al. used an information theoretical approach accounting for the time causality of the signal and the closeness centrality of different nodes. They discovered the pertinence of the alpha band while performing and distinguishing specific imaginary or visuomotor assignments.

Excitatory neurons in the visual cortex are significant in understanding brain functions. However, some neuron types and their morphological properties have not been fully deciphered. The authors in Zhang et al. applied the brain-wide positioning system to image the entire brain of two Thy1-eYFP $\mathrm{H}$-line male mice. Their results demonstrate a paradigm for resolving the visual cortex through single-neuron-level quantification and show potential to be extended to reveal the connectome of other sensory and motor systems.

Excessively high neural synchronization has been associated with epileptic seizures, one of the most common brain diseases worldwide. In Protachevicz et al., the authors studied neural synchronization in a random network where the dynamics on the nodes are given by adaptive exponential integrate-and-fire neurons with excitatory and inhibitory synapses. Their results demonstrate that external electrical stimulation not only can trigger synchronous behavior, but more importantly, can be used to reduce abnormal synchronization and thus, control epileptic seizures.

\section{REFERENCES}

1. Thurner S, Hanel R, Klimek P. Introduction to the theory of complex systems. Oxford, UK: (Oxford University Press (2018).

2. Barrat A, Barthelemy M, Vespignani A. Introduction to the modelling and analysis of complex systems. Cambridge, England: Cambridge University Press (2008).

3. Sayama H. Introduction to the Modelling and Analysis of Complex Systems. OPEN SUNY: Textbooks (2015).
Time-keeping in the medial premotor cortex is governed by four kinds of ramp cell populations. In Wei et al., a spiking neuron model is presented and used to construct a complete circuit for temporal processing. The authors showed that it can reflect many of the physiological neural circuits in the brain and explain some of the phenomena in the temporal-perception process.

Connectivity and biophysical processes determine the functionality of neural networks. The authors in Kim et al. developed a real-time framework, called Neural Interactome, to visualize simultaneously and interact with the structure and dynamics of neural networks. They examined scenarios studied experimentally, such as touch response circuits and explored new scenarios that did not undergo elaborate experimental studies.

Recurrent neural networks can produce sustained state-tostate transitions without any driving inputs, where their dynamical properties are determined by neural connection strengths. In Krauss et al., the authors used multi-dimensional scaling to study similarity relations among topologically distinct motifs. Strikingly, they reported that the key parameter that controls motif dynamics is the ratio of excitatory to inhibitory connections.

Finally, a new computational framework that implements asynchronous neural dynamics was used in Bronzon to address the duality between synchronous vs asynchronous processes and their relation to conscious vs unconscious behaviors. The new framework proposes a tool to study the emergence of brain structures that might be associated with higher level cognitive capabilities.

\section{AUTHOR CONTRIBUTIONS}

All authors listed have made substantial, direct, and intellectual contribution to the work and approved it for publication.

Conflict of Interest: The authors declare that the research was conducted in the absence of any commercial or financial relationships that could be construed as a potential conflict of interest.

Copyright (C) 2021 Antonopoulos, Rubido, Batista and Baptista. This is an open-access article distributed under the terms of the Creative Commons Attribution License (CC $B Y)$. The use, distribution or reproduction in other forums is permitted, provided the original author(s) and the copyright owner(s) are credited and that the original publication in this journal is cited, in accordance with accepted academic practice. No use, distribution or reproduction is permitted which does not comply with these terms. 